\title{
Autoconsciência e investimento esquemático da aparência em indivíduos com deficiência visual
}

\section{Self-consciousness and schematic investment of appearance in individuals with visual impairment}

\author{
José Mendes (1) \\ Durval Alcaidinho (2) \\ Maura Alcaidinho (3) \\ (1) INTELECTO - Psicologia \& Investigação, Ponta Delgada, Açores, Portugal \\ (2) Hospital Divino Espírito Santo, Ponta Delgada, Açores, Portugal \\ (3) Unidade de Saúde da Ilha de São Miguel, Ponta Delgada, Açores, Portugal \\ Recebido: 15/10/2018; Revisto: 21/12/2018; Aceite: 08/02/2019.
}

https://doi.org/10.31211/rpics.2019.5.1.99

\begin{abstract}
Resumo
Objetivo: A Autoconsciência da Aparência tem sido alvo de recente investigação em vários contextos, sendo desconhecidos estudos realizados em indivíduos com deficiência visual. Propõe-se com este estudo, compreender a Autoconsciência da Aparência em pessoas com deficiência visual. Método: Participaram 104 indivíduos (43 com cegueira congénita, 19 com baixa visão congénita, 23 com cegueira adquirida e 19 com baixa visão adquirida) que responderam a um conjunto de questionários quer disponibilizados on-line, quer de forma presencial, tendo sido adicionada a opção de resposta "não se aplica" em todos os itens da versão reduzida da Derriford Appearance Scale(DAS-24) e Appearance Scale Inventory(ASI-R).

Resultados: Todos os instrumentos utilizados apresentaram bons índices de consistência interna. Não se verificaram diferenças significativas no Investimento Esquemático e Autoconsciência da Aparência entre os tipos de deficiência visual. Os participantes com baixa visão congénita e baixa visão adquirida, apresentaram maior desconforto com a sua aparência, em comparação com os participantes com cegueira congénita e cegueira adquirida. O Afeto Negativo revelou-se preditor do investimento esquemático e autoconsciência da aparência. Conclusões: Preocupações da aparência em indivíduos com deficiência visual são generalizadas, ocorrendo internalização dos ideais do corpo também em sujeitos sem visão. O Afeto Negativo é um preditor do Investimento Esquemático e Autoconsciência da Aparência.
\end{abstract}

Palavras-Chave: Autoconsciência da Aparência; Deficiência Visual; Investimento Esquemático da Aparência; Afeto Positivo; Afeto Negativo.

\section{DI\&D | ISMT}

rpics@ismt.pt

https://rpics.ismt.pt
Publicação em Acesso Aberto

(C)2019. O(s) Autor(es). Este é um artigo de acesso aberto distribuído sob a Licença Creative Commons Attribution, que permite uso, distribuição e reprodução sem restrições em qualquer meio, desde que o trabalho original seja devidamente citado.
José Mendes

INTELECTO - Psicologia e Investigação. Rua do Monte, 52B, Fração L, R/C Esq.

9500-451, Ponta Delgada

Açores, Portugal

e-mail: josemendes@intelecto.pt 


\begin{abstract}
Aim: Self-consciousness of appearance has been recently subject to investigation in several contexts, and studies in visual impairment are unknown. The objective of this study is to understand the self-consciousness of appearance in visually impaired individuals. Method: 104 participants ( 43 with congenital blindness, 19 with congenital low vision, 23 with acquired blindness and 19 with acquired low vision) answered a set of questions administered online or face-to-face, with answers option (not applicable) in all questions of Derriford Appearance Scale-short (DAS-24) and Appearance Scale Inventory (ASIR). Results: All instruments used had good internal consistency. There were no significant differences in the investment in the physical appearance and self-consciousness of appearance between types of visual impairment. Negative Affect revealed to be a predictor of schematic investment and self-consciousness of appearance. Participants with congenital and acquired low vision were more uncomfortable with their appearance compared to participants with congenital and acquired visual impairment. Conclusion: Appearance concerns in the visually impaired are widespread, and internalization of body ideals also take place in blind individuals. Negative Affect is a predictor of schematic investment and self-consciousness of appearance.
\end{abstract}

Keywords: Self-consciousness of Appearance; Visual Impairment; Schematic Investment of Appearance; Positive Affect; Negative Affect.

\title{
Introdução
}

Nas últimas décadas, a investigação em psicologia tem-se centrado na importância que a aparência exerce no quotidiano dos indivíduos (Mendes, Figueiras, Moreira, \& Moss, 2016; Mendes \& Pereira, 2018), existindo a necessidade de melhor compreender as características psicológicas subjacentes dos indivíduos conscientes da sua aparência (Mendes, 2017). Segundo Rumsey e Harcourt (2005), examinamos a nossa aparência milhares de vezes durante a nossa vida, em que as mudanças físicas do corpo, principalmente as da face, têm um poderoso efeito sobre a forma como nos observamos.

A maioria dos indivíduos apresentam preocupações com a sua aparência (Harris \& Carr, 2001), descrevendo-se o conceito da imagem corporal como uma figuração sobre como o indivíduo se sente em relação à sua aparência (Cash, 2004; El Ansari, Vodder Clausen, Mabhala, \& Stock, 2010; Grogan, 2017). No entanto, a imagem corporal nas pessoas com deficiência visual suscita várias questões, por exemplo, "O que os indivíduos cegos pensam sobre o seu próprio corpo? Os cegos estão preocupados com a sua aparência? Os cegos procuram o corpo ideal?" (Kaplan-Myrth, 2000, p. 280). Segundo Alves e Duarte (2008), as limitações na capacidade visual podem trazer consequências para o desenvolvimento da imagem corporal em indivíduos com deficiência visual.

A maioria dos indivíduos partilha do senso que as pessoas com cegueira não percecionam qualquer tipo de luz. Contudo, o termo cego é amplo e abrange indivíduos que não têm visão e indivíduos que têm alguma perceção de luz e cor, conseguem ler grandes impressões e podem ver imagens numa televisão de circuito fechado (Kaplan-Myrth, 2000). Segundo Pitano e Noal (2018, p. 131), os indivíduos com deficiência visual "impõem uma organização sensorial diferenciada em relação à construção, organização e estruturação dos saberes". Behrmann, Moscovitch e Winocur (1994) mencionam que embora tenha sido sugerido que as imagens mentais se assemelham à perceção real de um objeto ou evento e a perceção e a imagem compartilharem mecanismos representacionais e neurais comuns, ainda não está claro até que ponto a perceção visual e as imagens mentais usam os mesmos mecanismos. González, Benito e Veiga (2004) mencionam a existência de experiências sensoriais intencionadas, onde o conhecimento global dos objetos que proporciona a informação visual é substituído por informação auditiva ou táctil, isto é, o 
indivíduo com deficiência visual constrói imagens pela articulação da informação adquirida (Pitano \& Noal, 2018).

Desde a infância que a deficiência visual tem repercussões na aparência física (e.g., lavar-se, cuidar das partes do corpo, vestir-se), sendo estas repercussões minimizadas pelas aprendizagens ao longo do desenvolvimento (González et al., 2004).

Noutro sentido, é frequente os pais incentivarem tanto menos a prática de exercício físico dos filhos quanto maior for a sua deficiência visual (Stuart, Lieberman, \& Hand, 2006), o que é contraproducente pois uma criança com deficiência visual necessita de fortes interações entre objetos e pessoas de forma a conseguir desenvolver e a tomar uma consciência do mundo exterior (Kaodoinski \& Toniazzo, 2017). Uma revisão de literatura (Eiras, Amorim, Carmo, \& Russo, 2012) apresenta uma concordância de estudos sobre os indivíduos com deficiência visual formarem uma perceção corporal pela dependência que estes têm sobre o seu próprio corpo e o meio em que se inserem, onde as informações sensoriais e o relato verbal são considerados pontos chave na construção da imagem corporal. De realçar que a linguagem dos indivíduos com deficiência visual é representativa dos conceitos desenvolvidos a partir de informações construídas pelos sentidos, existindo "redes conceituais de cada conceito inferidas a partir de pontos convergentes e divergentes e os caminhos, ou recursos preceptivos, utilizados pelos cegos na formação de conceitos" (Nunes \& Lomônaco, 2008, p. 128).

Um estudo de cariz qualitativo, realizado por Kaplan-Myrth (2000), menciona que as pessoas com deficiência visual se preocupam com a aparência física e quase todos os participantes entrevistados para o respetivo estudo cuidam da sua aparência através de regimes pessoais tais como a maquilhagem, exercício físico e moda, enfatizando a necessidade da importância dos ideais de corpo de indivíduos considerados modelos. Em reforço desta ideia, segundo Smedema e McKenzie (2010), as pessoas com deficiência visual podem sentir-se mais confortáveis quando executam o seu trabalho através da internet, uma vez que estes são avaliados mais pela força das suas contribuições do que pela sua aparência ou deficiência visual.

Nazaré, Moreira e Canavarro (2010) consideram que a imagem corporal se compõe pela saliência autoavaliativa e saliência motivacional, em que a perceção da imagem corporal é definida pelo rigor que o indivíduo faz da sua forma, comparando-as com as suas características reais. No entanto, o contacto social exerce um papel preponderante nas relações sociais, níveis de autoconceito e autoestima dos indivíduos com deficiência visual (Alves \& Duarte, 2008).

A maioria das decisões das pessoas com deficiência visual são tomadas pelos familiares e grupo de pares sem deficiência visual, levando-os a não de participar em atividades (laborais, extralaborais) que influenciam uma menor autodeterminação, isolamento e solidão das pessoas com deficiência visual (Robinson \& Lieberman, 2004). No entanto, os valores culturais de beleza impostos pela sociedade parecem não exercer grande influência no processo de construção da imagem corporal em indivíduos com deficiência visual (Alves \& Duarte, 2008); contudo, é escassa a investigação sobre como os indivíduos com deficiência visual percebem o seu próprio corpo (Peres, Espírito-Santo, Espírito, Ferreira, \& Assis, 2015).

Eiras et al. (2012) defendem que a perceção adequada da imagem corporal é fundamental para que os indivíduos com deficiência visual possam integrar de forma confiante a sociedade. Assim, de forma a contribuir para uma melhor compreensão de como o indivíduo com deficiência visual se sente e comporta 
relativamente às preocupações com a sua aparência, definimos quatro objetivos orientadores. Em primeiro lugar, iremos analisar a fiabilidade das escalas de forma a garantir que as mesmas são válidas para administrar em indivíduos com deficiência visual. Posteriormente, analisaremos possíveis diferenças no investimento esquemático, autoconsciência da aparência e os afetos (como traço da personalidade) entre sujeitos com diferentes tipos de deficiência visual. Em terceiro lugar, procederemos à análise de possíveis relações entre as mesmas variáveis, tendo-se por último analisado se os afetos serão preditores do investimento esquemático e autoconsciência da aparência.

\section{Método}

\section{Procedimento}

O comprometimento visual em indivíduos inviabiliza a aplicação de testes no formato tradicional (Oliveira \& Nunes, 2015), no entanto, o cuidado na aplicação dos instrumentos utilizados no estudo permitiu que estes apresentassem uma fiabilidade apropriada ( $\alpha$ de Cronbach $\geq 0,70$ ), com valores considerados moderadamente elevados (Marôco \& Garcia-Marques, 2006).

Apresentou-se o presente estudo à Direção da Associação dos Cegos e Amblíopes de Portugal (ACAPO), tendo-se definido posteriormente que a melhor abordagem de solicitar a participação dos indivíduos seria disponibilizar os instrumentos organizados através do Google Docs, acompanhados do Consentimento Livre, Informado e Esclarecido da respetiva participação. O estudo foi divulgado na revista eletrónica da ACAPO.

Perante a pouca adesão ao tipo de recrutamento, a ACAPO solicitou apoio à Associação Promotora do Ensino dos Cegos, tendo a respetiva direção autorizado e informado os seus utentes da realização de um estudo de carácter voluntário. Nesta fase, procedeu-se à impressão dos instrumentos e respetivo Consentimento Livre, Informado e Esclarecido que foram aplicados presencialmente nas instalações da instituição. Foi reforçado, no momento da aplicação dos instrumentos, que a participação era anónima e voluntária, podendo desistir-se de participar em qualquer momento.

\section{Participantes}

Participaram neste estudo 104 indivíduos com uma média de idade 39,72 anos. Somente 39 participantes responderam ao questionário on-line através de uma aplicação informática própria para audição das questões. Os restantes 65 participantes responderam diretamente às questões lidas pelo investigador principal com as seguintes instruções: "Serão lidas algumas afirmações que deverá responder conforme o grau de concordância. Não são possíveis comentários sobre as afirmações de cada instrumento, somente será possível repetir a leitura das afirmações as vezes necessárias". 
Tabela 1

Caracterização da Amostra (\%)

\begin{tabular}{llc}
\hline Características & & $n(\%)$ \\
\hline \multirow{2}{*}{ Eexo } & Masculino & $66(63,5)$ \\
& Feminino & $38(36,5)$ \\
\hline & $1{ }^{\circ}$ ciclo & $3(2,9)$ \\
& $2{ }^{\circ}$ ciclo & $6(5,8)$ \\
& 3 . ciclo $^{\circ}$ & $24(23,1)$ \\
& Secundário & $44(42,3)$ \\
& Ensino Superior & $27(26,0)$ \\
\hline \multirow{3}{*}{ Estado Civil } & Solteiro(a) & $50(48,1)$ \\
& Casado(a) & $38(36,5)$ \\
& Divorciado(a) & $1(1,0)$ \\
& União de Facto & $15(14,4)$ \\
\hline \multirow{3}{*}{ Local de Residência } & Grande Meio Urbano & $59(56,7)$ \\
& Pequeno Meio Urbano & $26(25,0)$ \\
& Grande Meio Rural & $11(10,6)$ \\
& Pequeno Meio Rural & $8(7,7)$ \\
\hline & Cegueira Congénita & $43(41,3)$ \\
& Baixa Visão Congénita & $19(18,3)$ \\
& Cegueira Adquirida & $23(22,1)$ \\
& Baixa Visão Adquirida & $19(18,3)$ \\
\hline
\end{tabular}

Nota. $N=104$

\section{Instrumentos}

Procedeu-se à realização de um Questionário Sociodemográficocom o intuito de explorar as variáveis Sexo, Escolaridade, Estado Civil, Local de Residência, Tipo de Deficiência Visual, Escolaridade e Preocupações com a Aparência (Tabela 1).

Derriford Appearance Scale-short [DAS24; Carr, Moss, \& Harris (2005); traduzido por Moreira \& Canavarro, (2007) e validado por Mendes et al. (2016)]. Esta escala mede o constructo subjacente da autoconsciência da aparência, num total de 24 itens respondidos numa escala tipo Likert, com seis questões adicionais. Os 24 itens têm o objetivo de avaliar a forma como o indivíduo se sente e comporta relativamente à característica que o incomoda, sendo cotados através das seguintes opções de resposta: "0 = N/A (não aplicável), 1 = Nunca/Quase nunca, 2 = Às vezes, 3 = Frequentemente e 4 = Quase Sempre" para os itens 3, 5, 7, 9, 12, 13, 15, 18, 19, 21, 24 e "1 = Nada, 2 = Ligeiramente, 3 = Moderadamente e 4 = Extremamente" para os restantes itens. 
As seis questões adicionais descrevem-se: uma sobre a existência de algum aspeto da aparência que preocupa o indivíduo; três questões abertas e outras duas com a pretensão de avaliar até que ponto o aspeto da aparência do indivíduo causa dor/desconforto ou o limita na capacidade física no seu quotidiano. O estudo psicométrico da versão portuguesa (Mendes et al., 2016) apresenta bom índice de consistência interna ( $\alpha$ de Cronbach =0,91; $K M O=0,93$ ). Pontuações elevadas indicam maior preocupação com a aparência.

The Appearance Schemas Inventory - Revised [ASI-R; Cash, Melnyk, \& Hrabosky (2004); traduzido e validado por Nazaré et al. (2010)]. Este inventário avalia o investimento esquemático na aparência através de duas dimensões (saliência auto-avaliativa e saliência motivacional; Cash et al., 2004) numa escala de autorresposta tipo Likert (20 itens), considerando uma cotação que varia entre 1 ("Discordo fortemente") e 5 ("Concordo fortemente"). Através da ASI-R pode avaliar-se os esforços de um indivíduo na forma como este gera a sua atratividade física e aparência. Quanto mais elevado for este resultado, maiores são os níveis de investimento esquemático relativos à aparência. A versão portuguesa do instrumento (Nazaré et al., 2010) apresenta características psicométricas idênticas à versão original ( $\alpha$ de Cronbach $=0,89$ ). Pontuações elevadas indicam maior investimento esquemático da aparência.

Positive and Negative Affect Schedule [PANAS; Watson, Clark, \& Tellegen (1988); traduzido e validado por Galinha \& Pais-Ribeiro (2005)]. O PANAS avalia estados emocionais específicos, sendo composto por 20 emoções organizadas em duas subescalas: afetos positivos ( $\alpha$ de Cronbach $=0,86$ ) e afetos negativos ( $\alpha$ de Cronbach $=0,89)$ com uma correlação entre subescalas perto de zero $(r=-0,10)$. Na soma das respostas às 10 emoções positivas e 10 emoções negativas (individualmente variam entre 1 = "nada ou muito ligeiramente", 2 = "um pouco", 3 = "moderadamente", 4 = "bastante" e 5 = "extremamente") quanto maior a pontuação, maior a experiência afetiva (positiva/negativa) dos indivíduos.

\section{Análise Estatística}

Pretende-se avaliar a fiabilidade dos instrumentos através do alfa de Cronbach ( $\alpha$ ).

A significância do efeito do Tipo de Deficiência Visual sobre os níveis de Autoconsciência da Aparência, Saliência Auto-avaliativa e Saliência Motivacional foi avaliada com uma ANOVA para cada uma das variáveis dependentes, seguida do teste post-hocHSD de Tukey para um alfa de 0,05 e respetivo tamanho do efeito (TDE) (Espírito-Santo \& Daniel, 2015, 2018).

Recorreu-se ao coeficiente de correlação de Pearson ( $r$ ), para análise das relações entre as variáveis. Utilizou-se a regressão linear múltipla de forma a permitir a obtenção de um modelo parcimonioso com a possibilidade de predizer a Autoconsciência da Aparência, Saliência Auto-avaliativa e Saliência Motivacional em função das variáveis independentes (Afeto Positivo e Afeto Negativo). Através da estatística de Durbin-Watson avaliou-se o pressuposto de independência e o VIF (Variance Inflating Factor) inferior a 5 para diagnosticar a multicolinearidade (Marôco, 2010), considerando efeitos significativos para um valor de $p$ inferior a 0,05. 


\section{Resultados}

Após análise da fiabilidade das escalas, verificaram-se níveis de boa consistência interna para todos os instrumentos (ASI-R, $\alpha$ de Cronbach =0,87; Saliência Auto-avaliativa $\alpha$ de Cronbach $=0,86$; Saliência Motivacional $\alpha$ de Cronbach = 0,70); DAS-24, $\alpha$ de Cronbach =0,88; Afeto Positivo, $\alpha$ de Cronbach = 0,84 e Afeto Negativo, $\alpha$ de Cronbach $=0,86$ ] para o presente estudo.

Quanto ao efeito do tipo de deficiência visual sobre as variáveis em estudo, não se verificaram diferenças estatisticamente significativas e o TDE foi pequeno entre o Tipo de Deficiência Visual e a Saliência Autoavaliativa ( $g$ de Hedges $=0,297$ ), Saliência Motivacional ( $g$ de Hedges $=0,261$ ) e Autoconsciência da Aparência ( $g$ de Hedges $=0,271$ ). No entanto, os participantes com Baixa Visão Congénita e Baixa Visão Adquirida apresentaram maiores níveis de investimento esquemático e autoconsciência da aparência (Tabela 2).

\section{Tabela 2}

Diferenças em Relação às Variáveis Investimento Esquemático (ASI-R) e Autoconsciência da Aparência (DAS24) entre Participantes com Diferentes Tipos de Deficiência Visual

\begin{tabular}{|c|c|c|c|c|c|c|c|}
\hline \multirow[b]{2}{*}{ Tipo de Deficiência Visual } & \multirow[b]{2}{*}{$N$} & \multirow[b]{2}{*}{$M$} & \multirow[b]{2}{*}{$D P$} & \multicolumn{2}{|l|}{ ANOVA } & \multicolumn{2}{|c|}{$95 \%$ IC } \\
\hline & & & & $F(g l)$ & $p$ & Inferior & Superior \\
\hline Saliência Auto-avaliativa (ASI-R) & & & & $1,87(3)$ & 0,14 & & \\
\hline Cegueira Congénita & 43 & 35,07 & 8,91 & & & 32,33 & 37,81 \\
\hline Baixa Visão Congénita & 19 & 39,21 & 8,65 & & & 35,04 & 43,48 \\
\hline Cegueira Adquirida & 23 & 33,43 & 10,15 & & & 29,04 & 37,83 \\
\hline Baixa Visão Adquirida & 19 & 38,16 & 9,12 & & & 33,76 & 42,55 \\
\hline Saliência Motivacional (ASI-R) & & & & $1,61(3)$ & 0,19 & & \\
\hline Cegueira Congénita & 43 & 25,49 & 5,57 & & & 23,77 & 27,20 \\
\hline Baixa Visão Congénita & 19 & 27,68 & 5,20 & & & 25,18 & 30,18 \\
\hline Cegueira Adquirida & 23 & 26,00 & 4,57 & & & 24,02 & 27,97 \\
\hline Baixa Visão Adquirida & 19 & 28,21 & 5,23 & & & 25,68 & 30,73 \\
\hline Autoconsciência da Aparência (DAS-24) & & & & $1,50(3)$ & 0,22 & & \\
\hline Cegueira Congénita & 43 & 48,88 & 16,76 & & & 56,58 & 64,54 \\
\hline Baixa Visão Congénita & 19 & 54,11 & 14,49 & & & 60,90 & 72,88 \\
\hline Cegueira Adquirida & 23 & 44,61 & 12,72 & & & 53,75 & 65,11 \\
\hline Baixa Visão Adquirida & 19 & 47,42 & 11,86 & & & 59,84 & 72,89 \\
\hline
\end{tabular}

Nota. $N=104 ; M=$ médias; $D P=$ desvios-padrão; $F=$ estatística $\mathrm{F}$ da ANOVA; $g /=$ graus de liberdade; $p=$ nível de significância; IC = intervalo de confiança.

Após aplicação do teste $t$ de Student e ANOVA não se verificaram diferenças estatisticamente significativas nas variáveis Investimento Esquemático e Autoconsciência da Aparência entre os sexos, categorias do estado civil, tipos de local de residência, tipo de deficiência visual, preocupações com aparência e níveis de escolaridade. 
Conforme demonstrado na Tabela 3, a variável Saliência Auto-avaliativa apresentou uma correlação positiva e significativa com variável Saliência Motivacional $\left(R^{2}=37,2 \%\right)$, a variável Autoconsciência da Aparência $\left(R^{2}=44,9 \%\right)$ e a variável Afeto Negativo $\left(R^{2}=29,2 \%\right)$; enquanto que a variável Saliência Motivacional apresentou uma relação positiva e significativa com a variável Autoconsciência da Aparência $\left(R^{2}=9,6 \%\right)$ e com a variável Afeto Negativo $\left(R^{2}=6,2 \%\right)$.

\section{Tabela 3}

Correlações Bivariadas entre Saliência Auto-avaliativa, Saliência Motivacional, Autoconsciência da Aparência, Afeto Positivo e Afeto Negativo

\begin{tabular}{lccccc}
\hline & 1 & 2 & 3 & 4 & 5 \\
\hline 1. Saliência Auto-avaliativa & & $0,61^{* *}$ & $0,67^{* *}$ & $-0,08$ & $0,54^{* *}$ \\
2. Saliência Motivacional & & & $0,31^{* *}$ & 0,07 & $0,25^{*}$ \\
3. Autoconsciência da Aparência & & & & $-0,15$ & $0,33^{* *}$ \\
4. Afeto Positivo & & & & & 0,12 \\
5. Afeto Negativo & & & & & \\
$M$ & 36,03 & 26,50 & 48,63 & 33,08 & 21,58 \\
$D P$ & 9,30 & 5,28 & 14,82 & 7,45 & 7,26 \\
\hline
\end{tabular}

Nota. $N=104$

${ }^{*} p<0,05 ;{ }^{* *} p<0,01$

Ao analisar possíveis preditores, procedeu-se a uma regressão linear múltipla, que após validação dos pressupostos homogeneidade, distribuição normal e independência dos erros, identificou-se o Afeto Negativo como preditor significativo da Saliência Auto-avaliativa, Saliência Motivacional e Autoconsciência da Aparência. As variáveis independentes conseguiram explicar em média $29 \%$ da variação da Saliência Auto-avaliativa e 13\% da variação da Autoconsciência da Aparência (Tabela 4).

\section{Tabela 4}

Regressão Linear Múltipla das Variáveis Preditoras da Saliência Auto-avaliativa, Saliência Motivacional e Autoconsciência da Aparência

\begin{tabular}{lcccccc}
\hline Variável dependente e preditor & $B$ & $\beta$ & $t$ & $p$ & $F(d f)$ & $R^{2}$ \\
\hline Saliência Auto-Avaliativa & & & & & $22,32(2)$ & 0,29 \\
$\quad$ Afeto Positivo & $-0,18$ & $-0,14$ & $-1,72$ & 0,089 & & \\
$\quad$ Afeto Negativo & 0,71 & 0,55 & 6,62 & $<0,001$ & & \\
Saliência Motivacional & & & & & $3,34(2)$ & 0,04 \\
$\quad$ Afeto Positivo & 0,03 & 0,04 & 0,40 & 0,687 & & \\
$\quad$ Afeto Negativo & 0,18 & 0,24 & 2,49 & 0,015 & & \\
Autoconsciência da Aparência & & & & & $8,69(2)$ & 0,13 \\
$\quad$ Afeto Positivo & $-0,38$ & $-0,19$ & $-2,04$ & 0,044 & & \\
$\quad$ Afeto Negativo & 0,73 & 0,36 & 3,86 & $<0,001$ & & \\
\hline
\end{tabular}

Nota. $N=104$. 


\section{Discussão}

Partindo do pressuposto que os instrumentos aplicados não haviam sido utilizados em indivíduos com deficiência visual, o presente estudo permitiu verificar a sua aplicabilidade e fiabilidade, apresentando níveis de consistência interna aceitáveis (Marôco \& Garcia-Marques, 2006).

O presente estudo, apesar de não evidenciar diferenças estatisticamente significativas entre os tipos de deficiência visual a nível do investimento esquemático e da autoconsciência da aparência, os participantes com baixa visão congénita e baixa visão adquirida apresentaram maior investimento na sua imagem corporal e maiores constrangimentos com a aparência em relação aos participantes com cegueira congénita e cegueira adquirida, ainda que ainda que a magnitude da diferença seja considerada pequena (EspíritoSanto \& Daniel, 2015). Em apoio parcial do presente resultado, estudos apontam para diferenças nos níveis de satisfação corporal entre indivíduos com deficiência adquirida em comparação com indivíduos com deficiência visual congénita (Peres et al., 2015), existindo diferenças das vivências e experiências sobre a imagem corporal entre as pessoas com deficiência visual congénita e pessoas com deficiência visual adquirida (Kaplan-Myrth, 2000).

Apesar das diferenças médias entre os indivíduos com deficiência visual adquirida e deficiência visual congénita, os participantes revelam estar atentos ou valorizam a gestão da aparência (saliência motivacional); consideram que a sua aparência integra a sua identidade (saliência auto-avaliativa) (Nazaré et al., 2010) e apresentam um conjunto de avaliações sobre a sua própria aparência (Mendes et al., 2016; Mendes \& Pereira, 2018). De facto, estudos têm demonstrado que os indivíduos com deficiência visual sentem insatisfação com a imagem corporal (Peres et al., 2015) e apresentam níveis de satisfação com os seus atributos pessoais, onde a imagem do corpo e a aparência apresentam as melhores percentagens do domínio psicológico (Rebouças, Araújo, Braga, Fernandes, \& Costa, 2016).

De realçar que a não existência de diferenças estatisticamente significativas entre os níveis de preocupação com a aparência no que diz respeito ao investimento esquemático e autoconsciência da aparência pode estar relacionada com o facto de a autoconsciência da aparência em indivíduos cegos ser generalizada (Kaplan-Myrth, 2000). Esta autora menciona que as pessoas com deficiência visual absorvem os ideias corporais da sociedade, mesmo sem visão. Enquanto os indivíduos sem deficiência avaliam com base numa comparação direta entre os seus próprios corpos e o corpo "ideal", as pessoas com deficiência conservam as avaliações de si mesmas através de comparações indiretas e vagas.

Por outro lado, a falta de visão pode influenciar ou mesmo retirar o verdadeiro significado à palavra Aparência, uma vez que esta pode ser utilizada meramente por imitação; por exemplo, a falta de visão pode influenciar conceitos adquiridos ao longo da vida e apesar de estes não dependerem da escolarização, podem ser verbalizados meramente por imitação (Kaodoinski \& Toniazzo, 2017). Segundo Peres et al. (2015) a construção da imagem corporal em indivíduos com deficiência visual é influenciada por valores simbólicos.

A existência de uma relação significativa entre o Afeto Negativo e o Investimento Esquemático e a Autoconsciência da Aparência, poderá estar relacionado com o facto de um elevado Afeto Negativo poder refletir desprazer e mal-estar subjetivo (Galinha, Pereira, \& Esteves, 2014), podendo inferir-se que os participantes no presente estudo apresentam insatisfação e preocupações com a aparência. Segundo Mosquera, Fernandes, Grzelczak e Arruda (2014), o processo de formação da imagem corporal depende de 
informações auditivas, táteis e cinestésicas, em que os pais desempenham um papel importante no desenvolvimento da imagem corporal. As famílias têm uma forte influência no desenvolvimento da identidade das pessoas com deficiência visual (Kaplan-Myrth, 2000), em que a superproteção por parte de familiares e amigos das pessoas com deficiência visual, pode influenciar um apoio social negativo (Cimarolli \& Boerner, 2005).

O Afeto Negativo revela-se também como variável preditor da saliência auto-avaliativa e da autoconsciência da aparência. Sendo o afeto composto por respostas afetivas das pessoas, e o afeto negativo dizer respeito à insatisfação (Noronha, Martins, Campos, \& Mansão, 2015), os participantes do presente estudo sentem menor valorização e gestão da aparência, maior insatisfação com o corpo (Nazaré et al., 2010) e maior constrangimento com a aparência (Carr et al., 2005).

Atendendo às limitações do presente estudo, por exemplo, o baixo número de participantes e o controlo de variáveis externas na aplicação verbal dos instrumentos, Moss e Rosser (2012), defendem que a insatisfação corporal é um conceito mais amplo que a autoconsciência da aparência, na medida em que inclui a infelicidade relacionada aos aspetos não visíveis do corpo. Assim, estudos futuros poderão concentrar-se na diferenciação entre a insatisfação corporal e autoconsciência da aparência em pessoas com deficiência visual.

Conflito de interesses | Conflict of interest: nenhum | none.

Fontes de financiamento | Funding sources: nenhuma | none.

Contributos | Contributions: JM: Coordenador do estudo; Supervisão do estudo; Revisão de literatura; Análise estatística; Redação Integral do manuscrito. DAe MA: Recolha de dados; Revisão de literatura.

Agradecimentos | Acknowledgements: Os autores agradecem à Dra. Rita Pereira (Coordenadora da Ação Social) da Associação dos Cegos e Ambíopes de Portugal pela sua prestigiada colaboração na divulgação deste estudo.

\section{Referências}

Alves, M. L. T., \& Duarte, E. (2008). Imagem corporal e deficiência visual: Um estudo bibliográfico das relações entre a cegueira e o desenvolvimento da imagem corporal [Body image and visual impairments: A bibliographic review of relationships between blindness and the development of body image]. Acta Scientiarum. Human and Social Sciences, 302), 147-154. https://doi.org/10.4025/actascihumansoc.v30i2.1936

Behrmann, M., Moscovitch, M., \& Winocur, G. (1994). Intact visual imagery and impaired visual perception in a patient with visual agnosia. Journal of Experimental Psychology: Human Perception and Performance, 20(5), 1068-1087. https://doi.org/10.1037/0096-1523.20.5.1068

Carr, T., Moss, T., \& Harris, D. (2005). The DAS24: A short form of the Derriford Appearance Scale DAS59 to measure individual responses to living with problems of appearance. British Journal of Health Psychology, 102), 285-298. https://doi.org/10.1348/135910705X27613

Cash, T. (2004). Body image: Past, present, and future. Body Image, 1(1), 1-5. https://doi.org/10.1016/S17401445(03)00011-1

Cash, T., Melnyk, S., \& Hrabosky, J. (2004). The assessment of body image investment: An extensive revision of the appearance schemas inventory. International Journal of Eating Disorders, 35(3), 305-316. https://doi.org/:10.1002/eat.10264 
Cimarolli, V., \& Boerner, K. (2005). Social support and well-being in adults who are visually impaired. Journal of Visual Impairment \& Blindness, 999), 521-534. Retrieved from https://files.eric.ed.gov/fulltext/EJ718696.pdf

Eiras, L. F. G., Amorim, B. B., Carmo, N. M., \& Russo, M. M. (2012). Construção da imagem corporal em deficientes visuais [Construction of body image in the visually impaired]. Arquivos em Movimento, 8(2), 94-110. Retrieved from https://revistas.ufrj.br/index.php/am/article/view/9208/pdf_20

El Ansari, W., Vodder Clausen, S., Mabhala, A., \& Stock, C. (2010). How do I look? Body image perceptions among university students from England and Denmark. International Journal of Environmental Research and Public Health, 72), 583-595. https://doi.org/10.3390/ijerph7020583

Espírito-Santo, H., \& Daniel, F. (2015). Calcular e apresentar tamanhos do efeito em trabalhos científicos (1): As limitações do $p<0,05$ na análise de diferenças de médias de dois grupos [Calculating and reporting effect sizes on scientific papers (1): $p<0.05$ limitations in the analysis of mean differences of two groups]. Revista Portuguesa de Investigação Comportamental e Social, 1(1), 3-16. https://doi.org/10.7342/ismt.rpics.2015.1.1.14

Espírito-Santo, H., \& Daniel, F. (2018). Calcular e apresentar tamanhos do efeito em trabalhos científicos (3): Guia para reportar os tamanhos do efeito para análises de regressão e ANOVAs [Calculating and reporting effect sizes on scientific papers (3): Guide to report regression models and ANOVA effect sizes]. Revista Portuguesa de Investigação Comportamentale Social, 4(1), 43-60. https://doi.org/10.31211/rpics.2018.4.1.72

Galinha, I. C., \& Pais-Ribeiro, J. L. (2005). Contribuição para o estudo da versão portuguesa da Positive and Negative Affect Schedule (PANAS): II - Estudo psicométrico [Contribution to the study of the Portuguese version of the Positive and Negative Affect Schedule (PANAS): II - Psychometric study]. Análise Psicológica, 23(2), 219-227. https://doi.org/10.14417/ap.84

Galinha, I. C., Pereira, C. R., \& Esteves, F. (2014). Versão reduzida da escala portuguesa de afeto positivo e negativo - PANAS-VRP: Análise fatorial confirmatória e invariância temporal [Short-form of the Portuguese version of the positive and negative affect schedule - PANAS-Port-VRP: Confirmatory factorial analysis and temporal invariance]. Psicologia, 28(1), 53-65. https://doi.org/10.17575/rpsicol.v28i1.622

González, R. P., Benito, J. C., \& Veiga, P. D. (2004). Deficiencia visual. Aspectos conceptuales y repercusiones funcionales [Visual deficiency. Conceptual aspects and functional repercussions]. In J. C. Benito, P. D. Veiga, \& R. P. González (Coords.), Psicología y ceguera: Manual para la intervención psicológica en el ajuste a la deficiencia visual [Psychology and blindness: Manual for psychological intervention in the adjustment to visual impairment]. Madrid, Spain: Organización Nacional de Ciegos Españoles.

Grogan, S. (2017). Body Image: Understanding body dissatisfaction in men, women and children (3 ${ }^{\text {rd }}$ ed.). New York: NY: Routledge.

Harris, D., \& Carr, A. (2001). Prevalence of concern about physical appearance in the general population. British Journal of Plastic Surgery, 54(3), 223-226. https://doi.org/10.1054/bjps.2001.3550

Kaodoinski, F., \& Toniazzo, F. R. (2017). Deficiência visual, interação e desenvolvimento da linguagem [Visual impairment, interaction, and language development]. SCRIPTA, 21(41), 185-203. https://doi.org/10.5752/P.2358-3428.2017v21n41p185

Kaplan-Myrth, N. (2000). Alice without a looking glass: Blind people and body image. Anthropology \& Medicine, 73), 277-299. https://doi.org/10.1080/713650612

Marôco, J. (2010). Análise estatística com o PASW Statistics (ex-SPSS)[Statistical analysis with PASW Statistics (ex-SPSS)]. Pêro Pinheiro: ReportNumber.

Marôco, J., \& Garcia-Marques, T. (2006). Qual a fiabilidade do alfa de Cronbach? Questões antigas e soluções modernas? [How reliable is Cronbach's alpha? Old issues and modern solutions?]. Laboratório de Psicologia, 4(1), 65-90. Retrieved from http://publicacoes.ispa.pt/index.php/lp/article/viewFile/763/706

Mendes, J. C. S. (2017). Fatores determinantes no ajustamento psicológico ao desfiguramento facial adquirido: A importância da autorregulação, representação cognitiva, emoções e autoconceito [Determinants of psychological adjustment: The importance of self-regulation, cognitive representation, and self-concept] 
(Doctoral dissertation, ISPA - $\quad$ Instituto Universitário, Lisboa). https://doi.org/10.13140/RG.2.2.11192.44806

Mendes, J., Figueiras, M., Moreira, H., \& Moss, T. (2016). Análise fatorial da versão portuguesa da Escala de Avaliação da Aparência de Derriford (DAS-24) [Factorial validity of the Derriford Appearance Scale (DAS-24) Portuguese Version]. Psychology, Community \& Health, 5(1), 31-43. https://doi.org/10.5964/pch.v5i1.128

Mendes, J., \& Pereira, V. (2018). Versão portuguesa reduzida da Escala de Avaliação da Aparência de Derriford (DAS-14): Análise fatorial exploratória e confirmatória [Derriford Appearance Scale (DAS-14) short Portuguese version: Exploratory and confirmatory analyse]. Revista Portuguesa de Investigação Comportamental e Social, 4(2), 25-32. https://doi.org/10.31211/rpics.2018.4.2.79

Moreira, H., \& Canavarro, M. C. (2007). The Portuguese version of the Derriford Appearance Scale - 24. Unpublished manuscript, Departamento de Psicologia, Universidade de Coimbra, Portugal.

Mosquera, C., Fernandes, L., Grzelczak, M., \& Arruda, M. (2014). Orientação e mobilidade: A imagem corporal como pré-requisito [Orientation and mobility: Body image as prerequisite]. InCantare, 5, 7-19. Retrieved from http://periodicos.unespar.edu.br/index.php/incantare/article/view/259/pdf_7

Moss, T. P., \& Rosser, B. A. (2012). The moderated relationship of appearance valence on appearance self consciousness: Development and testing of new measures of appearance schema components. PLOS ONE, フ11), e50605. https://doi.org/10.1371/journal.pone.0050605

Nazaré, B., Moreira, H., \& Canavarro, M. C. (2010). Uma perspectiva cognitivo-comportamental sobre o investimento esquemático na aparência: Estudos psicométricos do Inventário de Esquemas sobre a Aparência (ASI-R) [Cognitive-behavioral perspective on schematic inversion in appearance: Psychometric studies of the Appearance Schemas Inventory (ASI-R)]. Laboratório de Psicologia, 8(1), 21-36. Retrieved from https://estudogeral.sib.uc.pt/bitstream/10316/14292/1/Vers\%C3\%A30\%20final.pdf

Noronha, A. P. P., Martins, D. F., Campos, R. R. F., \& Mansão, C. S. M. (2015). Relações entre afetos positivos e negativos e os cinco fatores de personalidade [Relationship between positive and negative affects and the big five]. Estudos de Psicologia, 202), 92-101. https://doi.org/10.5935/1678-4669.20150011

Nunes, S. S., \& Lomônaco, J. F. B. (2008). Desenvolvimento de conceitos em cegos congênitos: Caminhos de aquisição do conhecimento [Concept development in congenital blinds: Means for knowledge acquisition]. Revista Semestral da Associação Brasileira de Psicologia Escolar e Educacional (ABRAPEE), 12(1), $119-138$. https://doi.org/10.1590/S1413-85572008000100009

Oliveira, C. M., \& Nunes, C. H. S. S. (2015). Instrumentos para avaliação psicológica de pessoas com deficiência visual: Tecnologias para desenvolvimento e adaptação [Instruments for the psychological assessment of visually impaired people: Technologies for development and adaptation]. Psicologia: Ciência e Profissão, 35(3), 886-899. https://doi.org/10.1590/1982-3703001902013

Peres, R. J., Espírito-Santo, G., Espírito, F. R., Ferreira, N. T., \& Assis, M. R. (2015). Insatisfação com a imagem corporal entre pessoas com deficiência visual [Dissatisfaction with body image among visually impaired individuals]. Revista Brasileira de Ciências do Esporte, 374), 362-366. https://doi.org/10.1016/j.rbce.2015.08.013

Pitano, S. C., \& Noal, R. E. (2018). Cegueira e representação mental do conhecimento por conceitos: Comparação entre cegos congênitos e adquiridos [Blindness and mental representation of knowledge by concepts: Comparison between congenital and acquired blind people]. Educação Unisinos, 22(2), $128-137$. https://doi.org/10.4013/edu.2018.222.02

Rebouças, C., Araújo, M. M., Braga, F. C., Fernandes, G. T., \& Costa, S. C. (2016). Avaliação da qualidade de vida de deficientes visuais [Evaluation of quality of life of visually impaired]. Revista Brasileira de Enfermagem, 69(1), 72-78. https://doi.org/10.1590/0034-7167.2016690110i

Robinson, B. L., \& Lieberman, L. J. (2004). Effects of visual impairment, gender, and age on self-determination. Journal of Visual Impairment and Blindness, 98(60), 350-366. Retrieved from https://files.eric.ed.gov/fulltext/EJ683612.pdf

Rumsey, N., \& Harcourt, D. (2005). The psychology of appearance. Maidenhead, England; New York: Open University Press. 
Smedema, S. M., \& McKenzie, A. R. (2010). The relationship among frequency and type of internet use, perceived social support, and sense of well-being in individuals with visual impairments. Disability and Rehabilitation, 32(4), 317-325. https://doi.org/10.3109/09638280903095908

Stuart, M. E., Lieberman, L., \& Hand, K. E. (2006). Beliefs about physical activity among children who are visually impaired and their parents. Journal of Visual Impairment \& Blindness, 1004), 223-234. Retrieved from https://files.eric.ed.gov/fulltext/EJ735712.pdf

Watson, D., Clark, L., \& Tellegen, A. (1988). Development and validation of brief measures of positive and negative affect: The PANAS scales. Journal of Personality and Social Psychology, 54(6), 1063-1070. https://doi.org/10.1037/0022-3514.54.6.1063 